\title{
Research on the Explosion Venting Assessment for the Relative Closed Building
}

\author{
Changxing Ren ${ }^{1,2}$, Wentao Chen ${ }^{3}$ \\ ${ }^{1}$ Tianjin Fire Research Institute of the MPS, Tianjin 300381 \\ ${ }^{2}$ National Center for Fire Engineering Technology, Tianjin 3003 \\ ${ }^{3}$ Beijing Research Center of Urban System Engineering, Beijing 10089 \\ 相对封闭建筑物爆炸泄压能力评估研究 \\ 任常兴 ${ }^{1,2}$, 陈文涛 ${ }^{3}$ \\ 1 公安部天津消防研究所, 天津 300381 , 中国 \\ 2 国家消防工程技术研究中心, 天津 300381, 中国 \\ 3 北京城市系统工程研究中心, 北京 100089 , 中国
}

\begin{abstract}
It was introduced on the requirements of explosion venting and the determination method of venting area for the relative closed building, and the application condition and scope were described in detail. The big spray plant including some complex workpiece was simulated by the FLACS, and the influences of suspended celling on the explosion overpressure, flame development, shock wave spread and consequence destroy were analyzed and compared. Moreover, the capacity of explosion venting for the complex building was evaluated. This study has some reference value on the design of explosion venting for the explosion atmosphere building.
\end{abstract}

Keywords: building; explosion venting; venting area; venting formula constant

\section{摘要}

介绍了国内外规范对相对封闭建筑物爆炸 泄压的要求和泄压面积的确定方法, 明确 了各自的使用条件和应用范围。采用 FLACS 软件对放置复杂工件的大型喷涂车间建筑 物开展了数值模拟分析, 对比了吊顶对爆 炸超压、火焰传播和冲击波形成及破坏后
果的影响程度, 可有效评估复杂建筑空间 的爆炸泄压能力。该研究可为气体和粉尘 爆炸性环境建筑物的爆炸泄压设计提供参 考依据。

关键词: 建筑物; 爆炸泄压; 泄压面积; 泄压比

\section{1. 引言}

依据我国现行《建筑设计防火规范》 (以下简称 “建规”) 和《粉尘爆炸泄压指 南》, 形成爆炸性环境的建构筑物要设置足 够的泄压面积, 即采用爆炸泄压技术来减 缓爆炸后果导致的危害和损失。爆炸泄压 是一种相对经济的降低后果破坏程度的防 护措施, 主要与爆炸性环境中可燃物质 (可燃气体、可燃蒸气或雾滴、可燃粉尘 等) 爆炸性参数、泄爆开启压力和包围体 的抗懪强度等有关, 但也要采取隔离措施 防止爆炸传播。爆炸泄压常用可形成爆炸 性环境的容器、管道和建构筑物等, 如煤 粉仓、有爆炸危险的厂房等, 且可能带来 火焰和压力的危害, 并可能对环境造成影 响, 爆炸泄压设计及应用时应予以考虑。 目前, 建规规定有爆炸危险的厂房或厂房 内有爆炸危险的部位应设置泄压设施, 宜 采用轻质屋面板、轻质墙体和易于泄压的 门窗等, 泄压面积采用泄压比值进行计 
算, 没有规定比值的需要试验测定, 但未 给出测定方法给工程设计带来不便。粉尘 爆炸泄压指南主要应用于对容器、筒仓与 设备的泄压设计, 引入最大泄爆压力、开 启压力、粉尘爆炸指数等进行泄压面积的 计算, 但对于可燃粉尘爆炸性环境的厂房 的泄压面积计算不明确。国内关于可燃性 气体爆炸性环境泄压计算, 尚未有专门的 设计规范。贾军等人 ${ }^{[1]}$ 分析了煤粉仓减压后 的最大爆炸压力、防爆门静开启压力和泄 压面积的变化规律。王宝兴、经建生等人 ${ }^{[2-}$ ${ }^{3]}$ 开展了大量粉尘泄压实验, 提出了一种计 算泄压面积的推荐方法。李明等人 ${ }^{[4]}$ 对比了 国内外爆炸性厂房泄压面积的计算方法, 有助于工程项目的爆炸泄压设计。国外关 于爆炸泄压的规范主要有美国消防协会 NFPA68 $8^{[5]}$ 、德国 VDI $3673^{[6]}$ 和欧盟 EN14491 $1^{[7]}$ 等, 通常依照保护对象的耐爆能 力、泄压设施的静开启压力以及可燃物质 的爆炸性参数等进行计算、实验或模拟确 定。吴宗之等 ${ }^{[8]}$ 人给出了油气泄漏事故的可 接受风险标准, P.Middha ${ }^{[9]}$ 采用 FLACS 开 展了氢气爆炸的数值模拟研究, 并验证了 模拟的有效性。为此, 对于含有多种可燃 物爆炸性环境的大型相对封闭建构筑可采 用数值模拟的方法进行泄压设计和评估。

\section{2. 建筑物的爆炸泄压分析}

关于建筑物爆炸泄压面积的计算, 现 行建规采用泄压比经验公式确定, 给出了 常见可燃粉尘或可燃气体的泄压比规定 值。对于爆炸指数 $\leq 30 \mathrm{MPa} \cdot \mathrm{m} / \mathrm{s}$ 的 $\mathrm{S}_{\mathrm{t}-1}$ 和 $\mathrm{S}_{\mathrm{t}-2}$ 类粉尘给出了泄压比的最小取值, 对于爆 炸性非常强的 $\mathrm{S}_{\mathrm{t}-3}$ 类粉尘不得小于 0.110 , 与甲烷、丙酮、液化石油气等可燃液体或 气体物质相当。对于可燃气体, 仅给出典 型少数几种, 给工程设计及应用带来不 便。

\section{1 建筑物可燃气体爆炸环境泄压计算}

美国消防协会标准 NFPA68-2013 第 7 章给出了可燃气体 (蒸气) 爆燃过程的泄 压计算 ${ }^{[5]}$, 要求爆炸性环境场所的长径比 $\leqslant$ 5 。对于低强度封闭建筑物, 爆炸后泄压 $P_{\text {red }} \leqslant 0.5 \mathrm{bar}$ 时, 泄压面积 $A_{\mathrm{v} 0}$ 计算如下:

$$
\begin{aligned}
A_{v 0} & =A_{s} C P_{\text {red }}^{1 / 2} \\
C & =\frac{S_{u} \rho_{u} \lambda}{2 G_{u} C_{d}}\left[\left(\frac{P_{\max }+1}{P_{0}+1}\right)^{1 / \gamma_{b}}-1\right]\left(P_{0}+1\right)^{1 / 2}
\end{aligned}
$$

式中, $A_{v 0}$ 为泄压面积, $\mathrm{m}^{2} ; A_{s}$ 为内 表面积, $\mathrm{m}^{2} ; P_{\text {red }}$ 为减压后的最大爆炸压 力, bar-g; $C$ 为泄压常数; $S_{\mathrm{u}}$ 为气体混合 物层流燃烧速率, $\mathrm{m} / \mathrm{s} ; \rho_{u}$ 为质量密度,

$\mathrm{kg} / \mathrm{m}^{3} ; \lambda$ 为气体的紊流燃烧速率与层流燃 烧速率的比值; $G_{u}$ 为不燃烧气体的音速流 量, $230.1 \mathrm{~kg} / \mathrm{m}^{2} / \mathrm{s} ; C_{\mathrm{d}}$ 为泄压口流量系 数; $P_{\max }$ 为可燃气体最大爆炸压力, bar$\mathrm{g} ; P_{0}$ 为封闭空间的初始压力, bar-g; $\gamma_{b}$ 为 燃烧气体的热比, 1.1 1.2。

对于泄压设备, 其静开启压力 $P_{\text {stat }}$ 通 常不高于减压后的最大爆炸压力 $P_{\text {red }}$ 。若 $P_{\text {red }} \leq 0.1 \mathrm{bar}, P_{\text {stat }} \leq P_{\text {red }}-0.024 \mathrm{bar}$; 若 $P_{\text {red }}>$ $0.1 \mathrm{bar}, P_{\text {stat }}<0.75 P_{\text {red。 }}$ 对于高强度密封空 间, 当减压后最大爆炸压力 $P_{\mathrm{red}}>0.5 \mathrm{bar}$, 最小泄压面积的计算公式如下:

$$
\begin{gathered}
A_{v 0}=A_{s} \frac{1-\left[\left(P_{\text {red }}+1\right) /\left(P_{\max }+1\right)\right]^{1 / \lambda b}}{\left[\left(P_{r e d}+1\right) /\left(P_{\max }+1\right)\right]^{1 / \lambda b}-\delta} \frac{S_{u} \rho_{u}}{G_{u}} \frac{\lambda}{C_{d}} \\
\delta=\frac{\left[\left(P_{\text {stat }}+1\right) /\left(P_{0}+1\right)\right]^{1 / \lambda b}-1}{\left[\left(P_{\max }+1\right) /\left(P_{0}+1\right)\right]^{1 / \lambda b}-1}
\end{gathered}
$$

\section{2 建筑物粉尘爆炸环境泄压计算}

欧盟标准 EN 14491-2012 附录 D.2 给出 了建筑物粉尘爆燃过程的泄压计算, 见上 式 (1)。计算过程需要考虑空间的内表面 积 $A_{\mathrm{s}}$, 通常包括屋顶或天花板、墙壁、地 板等。同时, 不必考虑无法承受预期压力 的非结构内部隔离物, 忽略设备和被包裹 结构的内表面积, 但包括毗邻厂房的内表 面积 ${ }^{[7]}$ 。

$$
A_{v}=A_{v 0} / E_{f}
$$

式中, $A_{\mathrm{v}}$ 为几何泄压面积, $\mathrm{m}^{2} ; E_{\mathrm{f}}$ 为泄压效 率; $C$ 为泄压常数, 依据粉尘的爆炸性等级 $\mathrm{S}_{\mathrm{t}}-0 、 \mathrm{~S}_{\mathrm{t}}-1$ 和 $\mathrm{S}_{\mathrm{t}}-2$ 分别取值 0.0057 $\mathrm{MPa}^{0.5} 、 0.0082 \mathrm{MPa}^{0.5}$ 和 $0.0095 \mathrm{MPa}^{0.5}$ 。对于 非细长建筑物, 没有形状尺寸限制; 对于 细长建筑物, 其长径比不应超过 3 。对于爆 炸性猛烈的 $\mathrm{S}_{\mathrm{t}}-3$ 粉尘, 该公式不适用。 


\section{3 相对封闭建筑物爆炸泄压模拟}

对于含有多种可燃物质形态的爆炸性 环境场所, 如大型造船厂和飞机组装车间 的喷涂工艺厂房等，相对封闭空间面积较 大，且内置设备占据大部分体积空间，空 洞等比较多, 采用传统的泄压公式计算比 较困难。FLACS 软件是基于 CFD 技术开发 的集成气体扩散、燃烧和爆炸后果数值模 拟功能的软件, 具备二维扩散、燃烧和爆 炸数学模型不可比拟的优势和特征, 可以 用于大型复杂空间的爆炸泄压数值模拟评 估分析。如郭正位等人[10]对 $110 \mathrm{kV}$ 油浸式 变电站主变压器室的可燃气体爆炸泄压采 用了 FLACS 数值模拟, 并给出了泄压面积 的拟合公式。

可燃气体 (蒸气) 爆炸与气云边界约 束条件、空间阻塞程度、结构特征、点火 位置以及减缓措施的影响有关, 如泄压面 板、水喷淋、惰性气体等, 采用数值模拟 方法可客观演化爆炸发展过程及压力的时 空分布。结合建构筑物爆炸性环境实际工 况, 确定最差事故场景, 考虑不同的可燃 气体云团位置及不同的点火位置, 采用 FLACS 对混合气体爆炸后果进行模拟。通 过对比各工况条件下建构筑内表面泄压部 位的超压及冲量, 评估屋顶、吊顶、窗、 门及外墙的泄爆效果。通常认为泄压效果 达到预期泄爆压力的 $90 \%$ 及其以上为较 好， $70 \% \sim 90 \%$ 之间为一般， $70 \%$ 以下为较 差。

\section{3. 实例分析}

\section{1 工程概况}

某大型喷涂车间轴线尺寸分别为 $60 \mathrm{~m} \times 60 \mathrm{~m} \times 15 \mathrm{~m}$ 和 $60 \mathrm{~m} \times 60 \mathrm{~m} \times 12 \mathrm{~m}$ 的单层厂 房, 钢筋混凝土排架结构, 屋面采用彩色 压型镀铝锌钢板, 可以降低厂房自重。喷 涂工艺要求 $20^{\circ} \mathrm{C} \sim 23^{\circ} \mathrm{C}$, 采用 $0.6 \mathrm{~m} \times 0.6 \mathrm{~m}$ 耐 火极限不低于 $0.25 \mathrm{~h}$ 的轻质吊顶保温隔热。 依据现行建规第 3.6.2 和 3.6.3 条款规定, 有 爆炸性的乙类厂房应设置泄压设施, 作为 泄压设施的轻质屋面板和墙体的质量 $\leq 60 \mathrm{~kg} / \mathrm{m}^{2}$ 。车间屋顶面密度为 $60 \mathrm{~kg} / \mathrm{m}^{2}$ 、
$30 \mathrm{~kg} / \mathrm{m}^{2}$, 吊顶密度为 $4.5 \mathrm{~kg} / \mathrm{m}^{2} 、 12 \mathrm{~kg} / \mathrm{m}^{2}$ 。 对于该气体爆炸环境的相对封闭车间, 轻 质保温吊顶是否影响屋面正常泄压, 拟采 用数值模拟方法, 基于对爆炸冲击波作用 强度的分析和比较来评估爆炸泄压能力。

\section{2 数值模拟}

喷涂车间的主要可燃物质挥发的有机 溶剂蒸气主要成分为二甲苯、松香水、酮 类、醇类等, 其中二甲苯约占 $70 \%$, 生产 火灾危险性为乙类, 设计泄压比不低于 0.11 。主要模拟 4 类工况: 无吊顶两种密度 屋顶, $30 \mathrm{~kg} / \mathrm{m}^{2}$ 屋顶与不同密度吊顶。考虑 若气体沉积底部, 相对均匀分布气云形状 为 $60 \mathrm{~m} \times 60 \mathrm{~m} \times 1.2865 \mathrm{~m}$, 若位于局部区域拟 设定为 $30 \mathrm{~m} \times 30 \mathrm{~m} \times 5.146 \mathrm{~m}$ 。以净高 $12 \mathrm{~m}$ 场 景为例, 其中一模拟场景的截面火焰与超 压变化如下图 1 所示。可见, 爆炸云团点火 后冲击波从点火点开始向四周传播, 总体 来说压力及冲量与距离点火位置的距离成 反比, 压力最大位置位于工件内部, 最大 爆炸压力为 $1.045 \mathrm{MPa}$ 。这主要是因为工件 物结构复杂, 内部设有多个隔板且空间狭 小, 易引发火焰的加速并形成较强的冲击 波及较大压力。

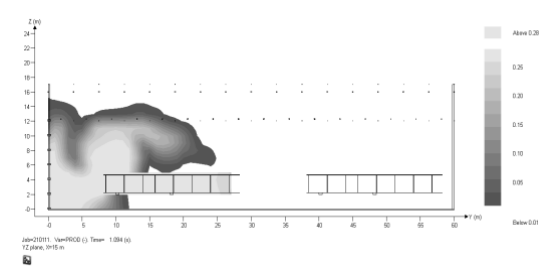

(a) 截面火焰

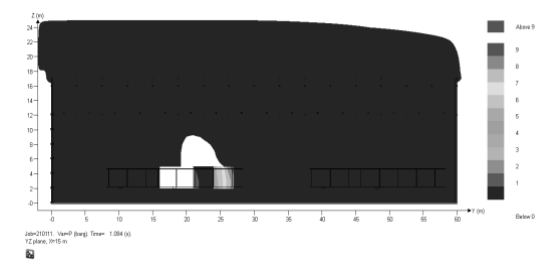

(b) 超压变化

图 1 某场景截面火焰与超压变化

\section{3 泄压效果评估}

采用 FLACS 软件模拟时在屋顶和喷涂 空间内不同位置设置测点, 比较有无吊顶 
工况场景的压力变化来分析吊顶对冲击波 到达屋顶时的压力影响, 从而评估吊顶存 在时对泄压效果的影响以及本身是否起到 明显的泄压作用。下图 2 为某测点在无吊顶 场景 (210111) 和有吊顶场景 (220111) 中 的压力对比, 可见吊顶的存在对冲击波波 形影响很小。

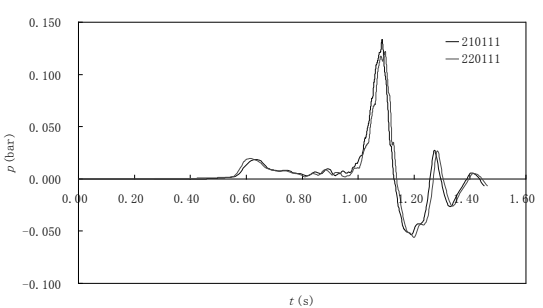

图 2 某测点位置不同场景超压变化曲线 对于有无吊顶场景的火焰发展和冲击

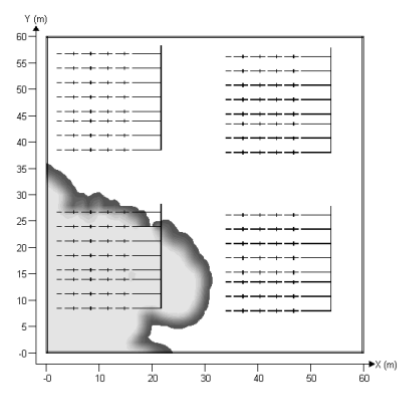

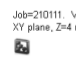

(a) 无吊顶
波形成比较如下图 3 和图 4 所示。可见, 爆 炸环境空间内吊顶对冲量的影响程度与云 团位置和点火位置有关, 根据模拟结果可 以判定设置吊顶增加了空间内的爆炸冲 量, 基本在 $10 \%$ 以内, 个别工况增大到 $15 \%$ 。

从可燃气云爆炸破坏效应角度分析 (超压破坏准则见下表 1 ), 以净高 $12 \mathrm{~m}$ 空 间场景为例, 有吊顶情况下, 对于破坏等 级 $\mathrm{B}(p \geq 0.35 \mathrm{ba})$ 的四个不同截面高度压力 变化如下图 5 所示。可见, 严重破坏的范围 相对较小, 主要分布在个别部分墙面和工 件开口部位。从总体模拟结果看, 由于厂 房内工件多空结构容易造成火焰加速, 形 成较强的冲击波, 整个车间都会处在中等 破坏等级, 严重破坏等级主要分布工件开 口部位, 与墙体应留有足够的间距。

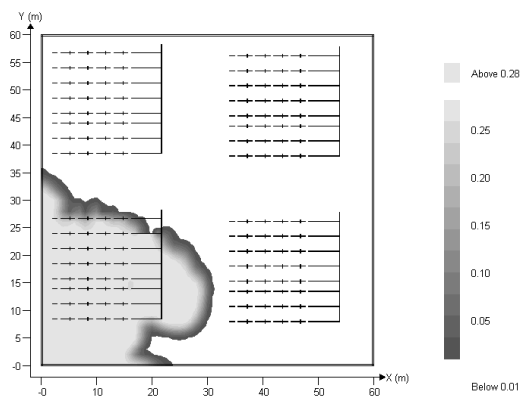

Jab $=220$
$x y$ plane
Bat

图 3 吊顶存在时 XY 截面火焰面发展对比

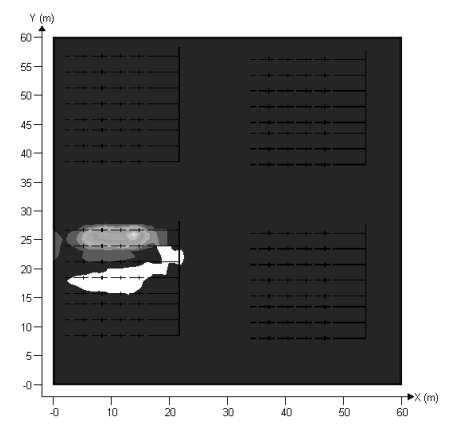

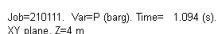

0
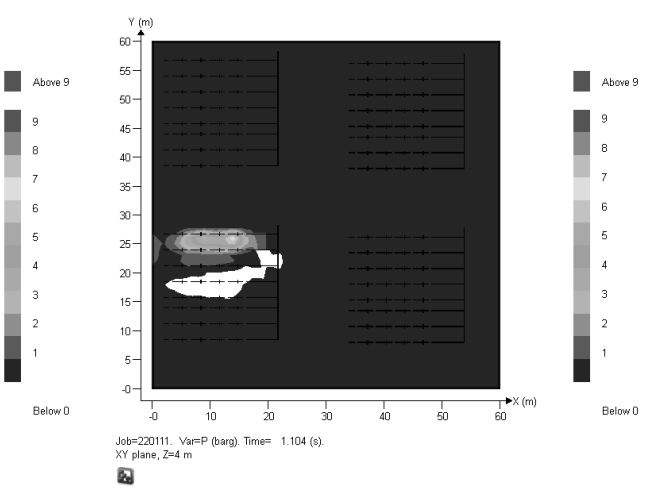

(b) 有吊顶

(a) 无吊顶

图 4 吊顶存在时 XY 截面超压发展对比 
表 1 超压破坏准则

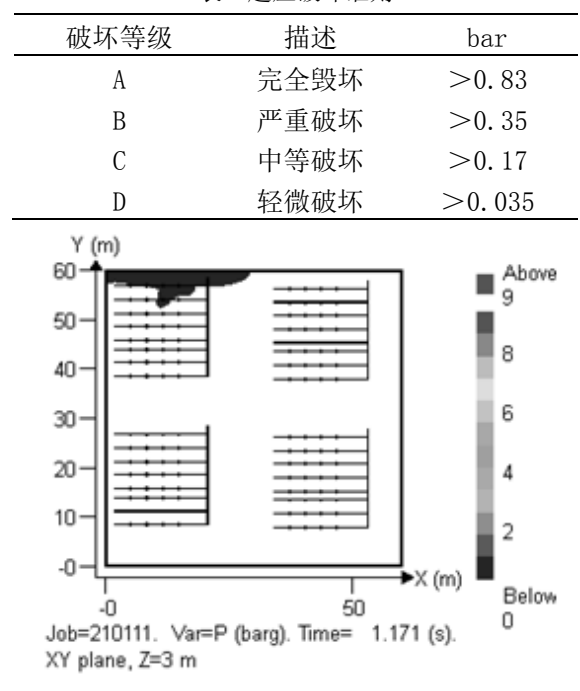

图 $5 \mathrm{XY}$ 截面严重破坏范围等值线图随时间的变化

\section{4. 结论}

(1) 相对封闭建筑物爆炸泄压可有效 降低后果破坏程度, 相对比较经济可行。 泄压效果主要与可燃物质的爆炸后果参数 (最大爆炸压力、爆炸指数和最大爆炸压 力上升速率等)、浓度分布、点火位置、泄 压设施的开启压力和包围体的抗爆强度等 有关。同时, 要采用爆炸隔离措施, 并考 虑泄压火焰和压力效应的危害。

(2) 建筑物泄压面积的计算主要有三 种方法: 建规泄压比经验公式法、基于泄 压后最大爆炸压力和可燃物燃爆特性参数 的确定模型、基于 FDS 的数值模拟法。建 规不能完全涵盖可燃气体物质, 缺少泄压 比参数确定方法; NFAP68 和 EN14491 计算 模型相对比较复杂, 考虑了可燃物的燃爆 特性和需要达到泄压能力, 均不适用猛烈 爆轰过程。数值模拟可以对建构筑物整体 的泄爆能力进行评估分析, 相对比较接近 实际工况, 适用于具有复杂建筑空间的场 景。

(3) 对于有吊顶的爆炸性环境建筑 物, 模拟结果表明: 泄压屋顶质量不变, 增加吊顶对冲击波形成和传播影响不大, 但可能增大爆炸超压和冲量值; 屋顶质量 不变, 增加吊顶质量可带来爆炸环境区内
部超压和冲量增大; 带多孔的工件, 可能 造成火焰加速形成较强爆炸冲击波, 开口 部位往往超压破坏严重。

\section{Acknowledgements}

This study was supported by Tianjin Key R\&D Program of China (No. 17YFZCSF00970).

\section{致谢}

本研究得到了天津市重点研发计划基金项 目 (17YFZCSF00970) 的资助。

\section{参考文献}

[1] 贾军, 任常兴, 李晋等. 煤粉仓泄爆抗爆 设计分析. 消防科学与技术, 2014, 33(3): 251-254.

[2] 王宝兴, 张银花, 马克辛. 粉尘爆炸泄压 研究. 工程热物理学报, 1992, 13(2):201206.

[3] 经建生, 王宝兴, 倪照鹏.一种计算泄压 面积的推荐方法. 消防科学与技术, 200 3, 22(3):177-180.

[4] 李明, 李超. 国内外有爆炸危险厂房的 泄压面积计算方法探讨. 科技经济导刊, 2016, (2):59-60.

[5] NFPA 68-2013. Standard on Explosion Protection by Deflagration Venting.

[6] VDI 3673. 1-2002. Pressure venting of dust explosions.

[7] EN 14491-2012. Dust explosion venting protective systems.

[8] Wu Z Z, Zhang S Z. Research progress on risk acceptance criteria of long distance oil and gas transportation pipeline. Journal of risk analysis and crisis response, 2012, 2(3):188-194

[9] Middha P. Development, Use and Validation of the CFD tool FLACS for Hydrogen Safety Studies. University of Burgen, Burgen, Norway, 2010.

[10] 郭正位, 刘湘莅, 郭新菊等. 油浸式变电 站主变压室泄压面积研究. 消防科学与 技术, 2015, 34(3): 303-306 